\title{
Cosmic ray acceleration and Balmer emission from SNR 0509-67.5
}

\author{
G. Morlino ${ }^{1}$, P. Blasi ${ }^{1,2}$, R. Bandiera ${ }^{1}$, and E. Amato ${ }^{1}$ \\ 1 INAF/Osservatorio Astrofisico di Arcetri, Largo E. Fermi 5, 50125 Firenze, Italy \\ e-mail: morlino@arcetri.astro.it \\ 2 INFN/Gran Sasso Science Institute, viale F. Crispi 7, 67100 L'Aquila, Italy
}

Received 28 June 2013 / Accepted 5 August 2013

\section{ABSTRACT}

\begin{abstract}
Context. Observation of Balmer lines from the region around the forward shock of supernova remnants may provide precious information on the shock dynamics and on the efficiency of particle acceleration at the shock.

Aims. We calculate the Balmer line emission and the shape of the broad Balmer line for parameter values suitable for SNR 0509-67.5, as a function of the cosmic ray acceleration efficiency and of the level of thermal equilibrium between electrons and protons behind the shock. This calculation aims to use the width of the broad Balmer line emission to infer the cosmic ray acceleration efficiency in this remnant.

Methods. We use the recently developed nonlinear theory of diffusive shock acceleration in the presence of neutrals. The semianalytical approach that we developed includes a description of magnetic field amplification as due to resonant streaming instability, the dynamical reaction of both accelerated particles and turbulent magnetic field on the shock, and all channels of interaction between neutral atoms and background plasma that change the shock dynamics.

Results. We achieve a quantitative assessment of the CR acceleration efficiency in SNR 0509-67.5 as a function of the shock velocity and different levels of electron-proton thermalization in the shock region. If the shock moves faster than $\sim 4500 \mathrm{~km} \mathrm{~s}^{-1}$, one can conclude that particle acceleration must be taking place with an efficiency of several tens of percent. For lower shock velocity the evidence of particle acceleration becomes less clear because of the uncertainty in the electron-ion equilibrium downstream. We also discuss the role of future measurements of the narrow Balmer line.
\end{abstract}

Key words. shock waves - ISM: supernova remnants - cosmic rays - acceleration of particles

\section{Introduction}

Supernova remnants (SNRs) are the most likely sources of the bulk of Galactic cosmic rays (CRs). Gamma ray observations carried out with both space-borne telescopes (AGILE, FermiLAT) and Cherenkov telescopes (MAGIC, HESS, VERITAS) are collecting a growing body of evidence of hadrons accelerated in SNRs, although fundamental questions still remain: direct evidence of CR acceleration up to the knee (in protons) is still missing, and the spectra of CRs as inferred from multifrequency observations appear to be somewhat softer than the basic predictions of the theory of diffusive shock acceleration (DSA). The Tycho SNR is currently the only instance of a young SNR in which the combined radio, X-ray, and gamma ray observations appear to be unambiguously explained as the result of acceleration of protons up to an energy close to the "knee" (Morlino \& Caprioli 2012).

Particle acceleration at SNR shocks is described by the nonlinear theory of diffusive shock acceleration (NLDSA; see Malkov \& Drury 2001, for a review). The formalism introduced by Amato \& Blasi $(2005,2006)$ and Caprioli et al. (2008) allows one to take into account the dynamical reaction of accelerated particles on the shock and the magnetic field amplification as due to streaming instability induced by the same accelerated particles. Conservation of momentum and energy leads to the straightforward prediction that, in the presence of efficient particle acceleration, the temperature of the plasma downstream of the shock should be lower than in the case where no acceleration takes place. Moreover, the dynamical reaction of accelerated particles on the background plasma induces the formation of a precursor upstream of the subshock.

The acceleration efficiency typically required for SNRs to be the sources of CRs is $\sim 10 \%$. In the pioneering paper by Chevalier \& Raymond (1978), it was first suggested that if the supernova explosion occurs in a partially ionized medium, the observation of the Balmer line width in proximity of the collisionless shock that develops can provide information on the temperature of the plasma and therefore on the efficiency of CR acceleration. More specifically, the broad Balmer line is expected to be narrower, reflecting a lower proton temperature downstream, and the narrow Balmer line is expected to be broader, reflecting heating in the precursor.

Despite the simple expectations illustrated above, a comprehensive description of the behavior of a collisionless supernova shock in the presence of neutrals, for arbitrary shock speed, has only recently been put forward. A first effort to include the presence of neutrals in the shock acceleration theory was made by Wagner et al. (2009) using a two-fluid model to treat ions and CRs but neglecting the dynamical role of neutrals. A different model was proposed by Raymond et al. (2011): here momentum and energy transfer between ions and neutrals is included, but both the CR spectrum and the profile of the CR-precursor are assumed a priori rather than calculated self-consistently. Blasi et al. (2012) propose a semi-analytical kinetic calculation that returns the distribution function of neutral hydrogen atoms in the absence of accelerated particles and all thermodynamical properties of the ion plasma. In that paper it was shown that a substantial fraction of neutral atoms that suffer a charge 
exchange (CE) reaction downstream produce neutral atoms that move towards upstream and can release energy and momentum in the upstream plasma, thereby heating it. Morlino et al. (2012) show that this phenomenon of neutral return flux creates an intermediate component of the Balmer line. Both these calculations were later used by Morlino et al. (2013a) to generalize the theory of NLDSA to include the effect of neutrals. This theory allows one to calculate the spectra of accelerated particles, the magnetic field amplification at the shock and the modifications induced on the shock by neutral atoms. The processes of CE and ionization, as well as excitation of atoms as due to collisions with both ions and electrons, are included in the calculations: all the elements are provided to accurately calculate the Balmer emission, as discussed in the article by Morlino et al. (2012).

Application of this detailed modeling to individual SNRs in which the strength and shape of the Balmer line are accurately measured may finally provide quantitative information on the efficiency of particle acceleration in these sources. In SNR 0509-67.5, a Balmer-dominated shock was already discovered in the 80's (Tuohy et al. 1982). Initially no broad Balmer line was detected because of the very low flux level, and detection of the broad component only occurred with the work by Ghavamian et al. (2007). These authors measured the width of the broad component of the $\mathrm{Ly} \beta$ line to be $3700 \pm 400 \mathrm{~km} \mathrm{~s}^{-1}$. However, as the spectrum was taken from the entire remnant, it remains uncertain whether the obtained line width is broadened by the bulk motion of the plasma. Only recently Helder et al. (2010) have been able to carry out a measurement of the broad component of the $\mathrm{H} \alpha$ line emission in two different regions of the blast wave, located in the southwest (SW) and northeast (NE) rim, obtaining a full width at half maximum (FWHM) of $2680 \pm 70 \mathrm{~km} \mathrm{~s}^{-1}$ and $3900 \pm 800 \mathrm{~km} \mathrm{~s}^{-1}$, respectively. The shock velocity was estimated to be $V_{\mathrm{sh}}=6000 \pm 300 \mathrm{~km} \mathrm{~s}^{-1}$ when averaged over the entire remnant, and $6600 \pm 400 \mathrm{~km} \mathrm{~s}^{-1}$ in the NE part. The width of the broad Balmer line was claimed by the authors to be suggestive of efficient CR acceleration.

The most important sources of uncertainty in deriving the CR acceleration efficiency for this SNR (and also in general) derive from the poor knowledge of the bulk speed of the forward shock and of the level of electron-ion thermalization behind the shock front. To extract information on CR acceleration from the measurement of the Balmer line, a theory is needed that accounts for the many subtleties of the modifications induced by both CRs and neutral atoms on the structure of a collisionless shock. Here we use the theoretical framework of Morlino et al. (2013a) to give a quantitative assessment of the evidence for CR acceleration in SNR 0509-67.5.

The paper structure is as follows. In Sect. 2 we discuss the existing estimates of the shock velocity and the way these estimates compare with the results of a simple model of the dynamics of this remnant. The results of our calculations, including the dynamical reaction of accelerated particles and the shock modification induced by neutrals, are illustrated in Sect. 3. In Sect. 4 we briefly discuss some predictions on the width of the narrow Balmer line in SNW 0509-67.5, with and without particle acceleration. We summarize in Sect. 5.

\section{Remnant dynamics}

At present, we are not aware of any direct measurement of the proper motion of the shock in SNR 0509-67.5. Helder et al. (2010) estimate the shock velocity by comparing the measured width of X-ray lines produced by shocked ejecta with the expectation of an evolutionary model of both the forward and reverse shock. These authors obtain $V_{\mathrm{sh}}=6000 \pm 300 \mathrm{~km} \mathrm{~s}^{-1}$ for the entire remnant, while they give an estimate of $5000 \mathrm{~km} \mathrm{~s}^{-1}$ for the SW region.

The shock speed can also be estimated from measurements of the SNR age and shock radius. SNR 0509-67.5 is located in the LMC, so that its distance from the Sun is known to be $50 \pm 1 \mathrm{kpc}$. The age estimated from the light echo is $400 \pm 120 \mathrm{yr}$ (Rest et al. 2005), although the spectral and dynamical properties of the SNR, together with some historical considerations, constrain the value to $400 \pm 50 \mathrm{yr}$ (Badenes et al. 2008). This remnant originated from a type Ia $\mathrm{SN}$, probably produced by a double-degenerate system (Schaefer \& Pagnotta 2012). Based on the high quality of the light echo, Rest et al. (2007) conclude that the parent event of SNR 0509-67.5 belonged to the group of overluminous, highly energetic type Ia $\mathrm{SNe}$, whose prototype is SN 1991Ts. Interestingly enough the same conclusion has been reached by Badenes et al. (2008) based on the X-ray spectrum: their best model has a kinetic energy of $1.4 \times 10^{51} \mathrm{erg}$ and a mass density of the circumstellar medium $\rho_{0}=10^{-24} \mathrm{~cm}^{-3}$.

At first sight the dynamics of 0509-67.5 may be constrained by two pieces of observational evidence. The first is the angular radius measured by Chandra in the X-ray band $\left(14.8^{\prime \prime} \pm 0.05^{\prime \prime}\right.$; Warren \& Hughes 2004), which translates into a linear radius of $1.1 \times 10^{19} \mathrm{~cm}(3.6 \mathrm{pc})$ at the known distance of the LMC. These measurements can be considered very accurate, because the errors on both angular radius and distance are only at the few percent level. Moreover Badenes et al. (2007) conclude that the expansion does not occur in a cavity, as would be expected for a type II SN explosion, but rather in the normal interstellar medium (ISM).

On the other hand, a more careful look at the morphology of this SNR shows that its shape deviates appreciably from spherical. The NE limb looks remarkably circular (see, e.g., the $\mathrm{H} \alpha$ image in the paper by Helder et al. 2010). By fitting a circle to the portion of the limb with a polar angle ranging from 20 to 140 degrees, we derive a mean radius of 16.1 arcsec (namely $3.9 \mathrm{pc}$ ) on that side, with average deviations less than 0.07 arcsec from that curve. However, assuming that the center of this circle coincides with the physical location of the supernova explosion, the distance to the SW limb can be estimated to be 13.2 arcsec (corresponding to $3.2 \mathrm{pc}$ ).

Even though these estimates depend on the conjecture that deviations from the spherical symmetry are more prominent on the SW side, this appears at least qualitatively reasonable given the strong enhancement on that side of dust emission, seen both with Spitzer (Borkowski et al. 2006) and with AKARI (Seok et al. 2008), also accompanied by brighter X-ray limb emission (Warren \& Hughes 2004) and Balmer emission. Moreover, the $\mathrm{H} \alpha$ line emission in the SW limb shows a higher value of the ratio $I_{\mathrm{b}} / I_{\mathrm{n}}$ compared with the NE region, compatible with a lower shock velocity.

Here we try to identify the range of meaningful values of the shock velocity in the two regions by modeling the dynamics of SNR0509-67.5 according to the analytical description of Truelove \& McKee (1999) for the evolution of a remnant expanding into a uniform medium with density $\rho_{0}$. Following Badenes et al. (2008), we fix the mass of the ejecta to the standard value $M_{\mathrm{ej}}=1.4 M_{\odot}$. The structure function of the ejecta is taken as $\propto\left(v / V_{\mathrm{ej}}\right)^{-7}$ (see Sects. 3.2 and 9 in Truelove \& McKee 1999). The explosion energy $E_{\mathrm{SN}}$ is taken as a free parameter. For each value of $E_{\mathrm{SN}}$ there is only one value of $\rho_{0}$ for which the calculated radius matches the observed value. In Fig. 1 we plot the shock velocity as a function of the SN energy for a SNR radius of $3.6 \mathrm{pc}$ (the average over the whole remnant) in the upper 

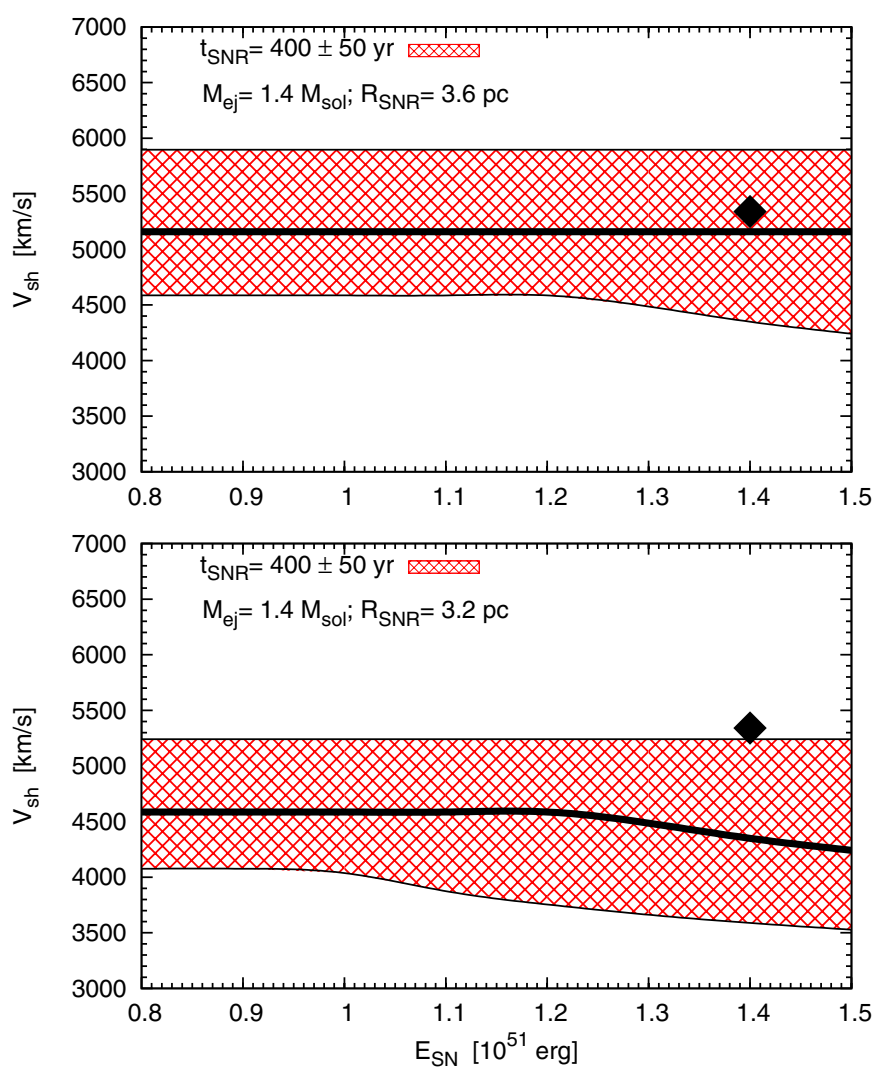

Fig. 1. Velocity of the forward shock as a function of explosion energy calculated using the theoretical model of Truelove \& McKee (1999) for a shock expanding into a uniform medium with density $\rho_{0}$, and assuming a power law density profile for the ejecta with power law index $n=7$. The upper (lower) panel refers to a SNR radius $R_{\mathrm{SNR}}=3.6 \mathrm{pc}$ $\left(R_{\mathrm{SNR}}=3.2 \mathrm{pc}\right)$. The thick curve is obtained matching the observational constraints derived from the remnant radius ( $3.6 \mathrm{pc}$ for the upper panel and $3.2 \mathrm{pc}$ for the lower panel) and age $(400 \pm 50 \mathrm{yr})$. The mass of the ejecta is fixed at $M_{\mathrm{ej}}=1.4 M_{\odot}$. The hatched area shows the results allowing for the uncertainty in the age of the remnant (the lower side corresponding to $450 \mathrm{yr}$ while the upper to $350 \mathrm{yr}$ ). The filled diamond shows the shock velocity calculated using the best fit parameters obtained by Badenes et al. (2008); i.e., $E_{\mathrm{SN}}=1.4 \times 10^{51} \mathrm{erg}$ and $\rho_{0}=10^{-24} \mathrm{~g} \mathrm{~cm}^{-3}$.

panel and $3.2 \mathrm{pc}$, probably more appropriate to the SW rim, in the lower panel. In both panels the hatched area illustrates the uncertainty in the age of the remnant. The diamond represents the shock velocity calculated using the best fit parameters obtained by Badenes et al. (2008); i.e. $E_{\mathrm{SN}}=1.4 \times 10^{51} \mathrm{erg}$ and $\rho_{0}=10^{-24} \mathrm{~cm}^{-3}$, which give $V_{\mathrm{sh}}=5340 \mathrm{~km} \mathrm{~s}^{-1}$. One should, however, keep in mind that this estimate was obtained for the whole remnant, while the relevant velocity for our calculations is the local shock speed in the region where the Balmer emission is measured. In fact, for the $\mathrm{SW} \operatorname{rim}\left(R_{\mathrm{SNR}}=3.2 \mathrm{pc}\right)$ one can see that shock velocities as low as $\sim 3500 \mathrm{~km} \mathrm{~s}^{-1}$ are still marginally compatible with the dynamics of this SNR.

To take the uncertainty in $V_{\text {sh }}$ into account, reflecting the different results found by Badenes et al. (2008), Helder et al. (2010) and our own calculations, we calculate the Balmer emission for $V_{\mathrm{sh}}=4000$ and $5000 \mathrm{~km} \mathrm{~s}^{-1}$ for the $\mathrm{SW}$ rim and for $6000 \mathrm{~km} \mathrm{~s}^{-1}$ for the NE rim. Calculations of the Balmer emission are also affected rather heavily by uncertainties in the electronion thermalization downstream of the shock. For the values of gas density typical of the ISM, the SNR shock is collisionless and electrons and protons are not expected to equilibrate rapidly to the same temperature. While Coulomb scattering is usually far too slow to bring electrons and protons into thermal equilibrium, some collective effects may lead to at least some partial balance, though our poor knowledge of the processes at work makes it impossible to assess the issue quantitatively. Below, we use the ratio $\beta_{\text {down }}$ of electron to proton temperature as a parameter, while phenomenological hints to its value can be found in the literature.

By modeling the X-ray spectra of shocked ejecta, Badenes et al. (2008) suggest that the allowed range of values for $\beta_{\text {down }}$ is $0.01<\beta_{\text {down }}<0.1$. Although this result strictly applies to the reverse shock, for this SNR the speed of the latter is expected to be not much lower than that of the forward shock, based on evolutionary models. Therefore a similar range could be expected for the outer shock. If so, electrons and protons appear to be rather far from thermal equilibrium.

As a more general statement, the current literature, which is based on various techniques, seems to be converging on a consensus that the level of equilibrium between electrons and protons lowers with increasing shock velocity (see Rakowski 2005, for a review). As a conservative estimate, for $V_{\mathrm{sh}}>3000 \mathrm{~km} \mathrm{~s}^{-1}$ one can take $T_{\mathrm{e}} / T_{\mathrm{p}} \lesssim 0.2$ (see fig. 6 in Rakowski 2005). A comprehensive review of the problem of electron-ion balance in collisionless shocks and the role of Balmer line measurements has recently appeared in the literature (Ghavamian et al. 2013).

Special caution is needed in using these estimates to infer the efficiency of particle acceleration., because some of them are derived by making use of the measured intensity ratio of broad to narrow Balmer lines. This quantity is actually also sensitive to the efficiency of particle acceleration, so that the information it contains becomes degenerate when this process is important.

\section{FWHM of the broad Balmer line and $C R$ acceleration}

The calculations of the shock structure of SNR 0509-67.5 were carried out by using the formalism of Morlino et al. (2013a), where both the CR back-reaction and the shock modification induced by neutral hydrogen are taken into account. As discussed by Morlino et al. (2013b), the kinetic approach adopted for the description of the neutral distribution function is crucial in this type of calculation: for fast shocks $\left(V_{\mathrm{sh}}>2500 \mathrm{~km} \mathrm{~s}^{-1}\right)$ neutral hydrogen atoms do not reach thermal equilibrium with the background ions despite the $\mathrm{CE}$ reactions that couple them together. This is due because ionization occurs before such equilibrium can be reached. As a consequence, the width of the Balmer line, which reflects the mean energy per particle of neutrals downstream of the shock, is somewhat less than predicted by assuming thermalization after a few CE reactions, as was done, for instance, by van Adelsberg et al. (2008). Since our kinetic calculations follow the evolution of the distribution function of neutral hydrogen in detail, we are able to predict the shape of the Balmer line very reliably, without any assumption of it being a Maxwellian.

In Fig. 2 we show the FWHM of the broad line as a function of shock speed, as obtained from our calculations in the absence of CR acceleration. The two data points represent the FWHM as measured by Helder et al. (2010) in the NE and SW regions of SNR 0509-67.5. As discussed above, the shock velocity is rather uncertain, which implies that the horizontal coordinate of the two points can actually be appreciably different from the one in the figure, which is taken directly from the work of Helder et al. (2010). The solid (dotted) line is the FWHM we predict in 


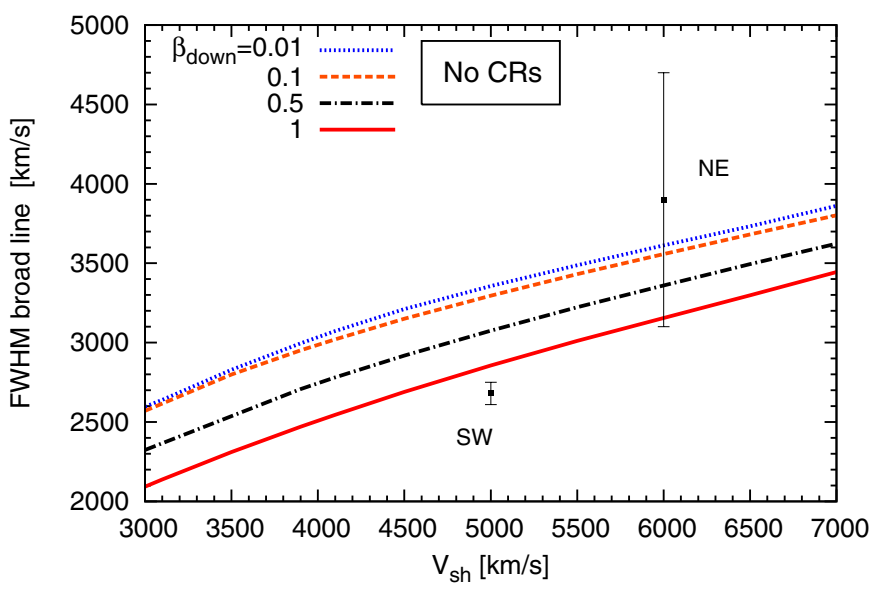

Fig. 2. FWHM of the broad line in the absence of CRs and for different levels of electron-ion equilibrium. The two data points represent the FWHM as measured by Helder et al. (2010) in the NE and SW regions of SNR 0509-67.5. Because the shock velocity is rather uncertain, the horizontal coordinate of the two points can actually be appreciably different from that in the figure, which is taken directly from the work of Helder et al. (2010).

the absence of CR acceleration and for $\beta_{\text {down }}=1\left(\beta_{\text {down }}=0.01\right)$. The large error bar on the FWHM in the NE rim makes it difficult to infer anything about particle acceleration in that region of the shock. On the other hand, in the SW region, the measured FWHM of the broad Balmer line is appreciably below the prediction, regardless of the level of electron-proton thermalization, if the shock velocity is higher than $\sim 4500 \mathrm{~km} \mathrm{~s}^{-1}$. This fact strongly suggests that $\mathrm{CR}$ acceleration may be taking place in the SW region of the remnant.

The calculations are therefore repeated for the SW rim, for two values of the shock velocity and by varying the CR acceleration efficiency. The $\mathrm{CR}$ acceleration efficiency is defined here as $\epsilon_{\mathrm{CR}}=P_{\mathrm{CR}} / \rho_{0, \text { tot }} V_{\mathrm{sh}}^{2}$, although another definition is more meaningful from the physical point of view: $\epsilon_{\mathrm{CR}}^{*}=P_{\mathrm{CR}} / \rho_{0 \text {, ion }} V_{\mathrm{sh}}^{2}$, where $\rho_{0 \text {,ion }}=\left(1-h_{\mathrm{N}}\right) \rho_{0 \text {,tot }}$ is the density of ionized material at upstream infinity, and $h_{\mathrm{N}}$ is the neutral fraction. One should keep in mind that collisionless shocks only act on the ionized component of the background fluid and that only ions can take part in the acceleration process.

The FWHM of the broad Balmer line as a function of $\epsilon_{\mathrm{CR}}$ (on the lower $x$-axis) and $\epsilon_{\mathrm{CR}}^{*}$ (upper $x$-axis) is reported in Fig. 3. The fraction of neutrals far upstream is taken as $h_{\mathrm{N}}=50 \%$ in the upper panels and $h_{\mathrm{N}}=10 \%$ in the lower panels. The two columns correspond to different values of the shock velocity, $V_{\text {sh }}=4000 \mathrm{~km} \mathrm{~s}^{-1}$ (on the left) and $V_{\text {sh }}=5000 \mathrm{~km} \mathrm{~s}^{-1}$ (on the right), which are expected to bracket the most likely interval of values of the shock velocity in the SW rim. The four curves in each panel refer to different levels of electron-ion thermalization, as specified in the figure. The shaded area in each panel shows the measured FWHM (Helder et al. 2010) with the $1 \sigma$ error bar.

As expected, the FWHM of the broad Balmer line decreases with increasing acceleration efficiency. The intersection between each line and the shaded area identifies the CR acceleration efficiency required to explain the data. For $V_{\mathrm{sh}}=5000 \mathrm{~km} \mathrm{~s}^{-1}$ and neutral fraction $h_{\mathrm{N}}=50 \%, \epsilon_{\mathrm{CR}}^{*}$ (top $x$-axis) ranges from $10-23 \%$, for the case of full equilibrium $\left(\beta_{\text {down }}=1\right)$, to $>57 \%$ for $\beta_{\text {down }}=0.01$. For the same value of the shock velocity and $10 \%$ neutral fraction, $\epsilon_{\mathrm{CR}}^{*}$ ranges from $\sim 10 \%$ for the case of full equilibrium $\left(\beta_{\text {down }}=1\right)$ to $\sim 35-40 \%$ for $\beta_{\text {down }}=0.01$. As expected, the evidence for $\mathrm{CR}$ acceleration becomes weaker when the shock velocity is lower: for $V_{\mathrm{sh}}=4000 \mathrm{~km} \mathrm{~s}^{-1}$ there is no evidence for particle acceleration if $\beta_{\text {down }}=1$ (full equilibrium), for any value of the ionization fraction. On the other hand, if $\beta_{\text {down }}=0.1-0.01$, the measured FWHM is still compatible with $\epsilon_{\mathrm{CR}}^{*} \simeq 20-30 \%$ for $h_{\mathrm{N}}=50 \%$ and $\sim 20 \%$ for $h_{\mathrm{N}}=10 \%$.

The calculation presented in Fig. 3 has been carried out assuming the best fit value for the upstream density inferred by Badenes et al. (2008), namely $n_{0}=0.6 \mathrm{~cm}^{-3}$. It is worth pointing out that in the absence of CR acceleration the predicted FWHM does not depend on the value of the density. For efficient CR acceleration the FWHM changes very slightly by changing the gas density: lowering the density to $0.1 \mathrm{~cm}^{-3}$, the FWHM changes only at the level of $\sim 1 \%$ for $\epsilon_{\mathrm{CR}}^{*}>50 \%$. For lower values of $\epsilon_{\mathrm{CR}}^{*}$, we find that the variation is totally negligible.

For the sake of completeness, we also calculated the Balmer emission expected from the NE rim, with $V_{\mathrm{sh}}=6000 \mathrm{~km} \mathrm{~s}^{-1}$. Our results are shown in Fig. 4 for $h_{\mathrm{N}}=50 \%$ (top panel) and $h_{\mathrm{N}}=10 \%$ (lower panel), compared with the FWHM measured by Helder et al. (2010). As expected, in this case we can only derive an upper limit on the acceleration efficiency: $\epsilon_{\mathrm{CR}}^{*}<50 \%$ for $h_{\mathrm{N}}=50 \%$ and $\epsilon_{\mathrm{CR}}^{*}<30 \%$ for $h_{\mathrm{N}}=10 \%$.

An estimate of the acceleration efficiency in the SW rim has also been presented by Helder et al. (2010), based on calculations previously carried out by van Adelsberg et al. (2008) and on generalized Rankine-Hugoniot relations including the pressure of accelerated particles and their escape. Such calculations of the width of the Balmer line were not based on self-consistent modeling of the nonlinear interactions that determine the shock structure: the shock modification due to neutrals and accelerated particles was only described in a phenomenological way. Moreover, as discussed in Morlino et al. (2013b), the calculations presented by van Adelsberg et al. (2008) are based on the assumption that the distribution function of neutral hydrogen atoms becomes Maxwellian, with the same temperature as for protons, for particles that have suffered more than two reactions of CE downstream. Our kinetic calculations show that this is not the case (see Morlino et al. 2013b, where the comparison with the results of van Adelsberg et al. 2008, was discussed in detail). The FWHM derived by van Adelsberg et al. (2008) is close to the results of our kinetic calculations only for shocks with $V_{\text {sh }}<2500 \mathrm{~km} \mathrm{~s}^{-1}$, but is systematically larger than our findings for faster shocks. This is due to the fact that for fast shocks, ionization occurs before a large number of CE reactions can drive ions and neutrals towards thermal equilibrium, so that the assumptions made by van Adelsberg et al. (2008) fail and a full kinetic calculation is required. By making a simple linear extrapolation of the curves in van Adelsberg et al. (2008), Helder et al. (2010) derive a lower limit on the acceleration efficiency of $>15 \%$, obtained for full electron-ion balance. Given the limitations of such a simple approach, this lower limit can be considered in reasonably good agreement with our more detailed determination of the acceleration efficiency, if the shock velocity is assumed to be $V_{\mathrm{sh}}=5000 \mathrm{~km} \mathrm{~s}^{-1}$, as in Helder et al. (2010). Given the uncertainties in the shock velocity, this bound should not be taken as an absolute lower limit.

As discussed above, in the case when electrons and ions downstream are close to full equilibrium $\left(\beta_{\text {down }} \sim 1\right)$, we find that the data are compatible with the absence of particle acceleration if the shock velocity is below $\sim 4500 \mathrm{~km} \mathrm{~s}^{-1}$, a value that still fits well with models for the dynamical evolution of the SNR (see Fig. 1). The rapid electron-ion equilibrium required, however, is somewhat at odds with the expectation for a fast collisionless shock, although it cannot be ruled out as yet. On the other hand, if the equilibrium level were low $\left(\beta_{\text {down }} \lesssim 0.1\right)$, then 

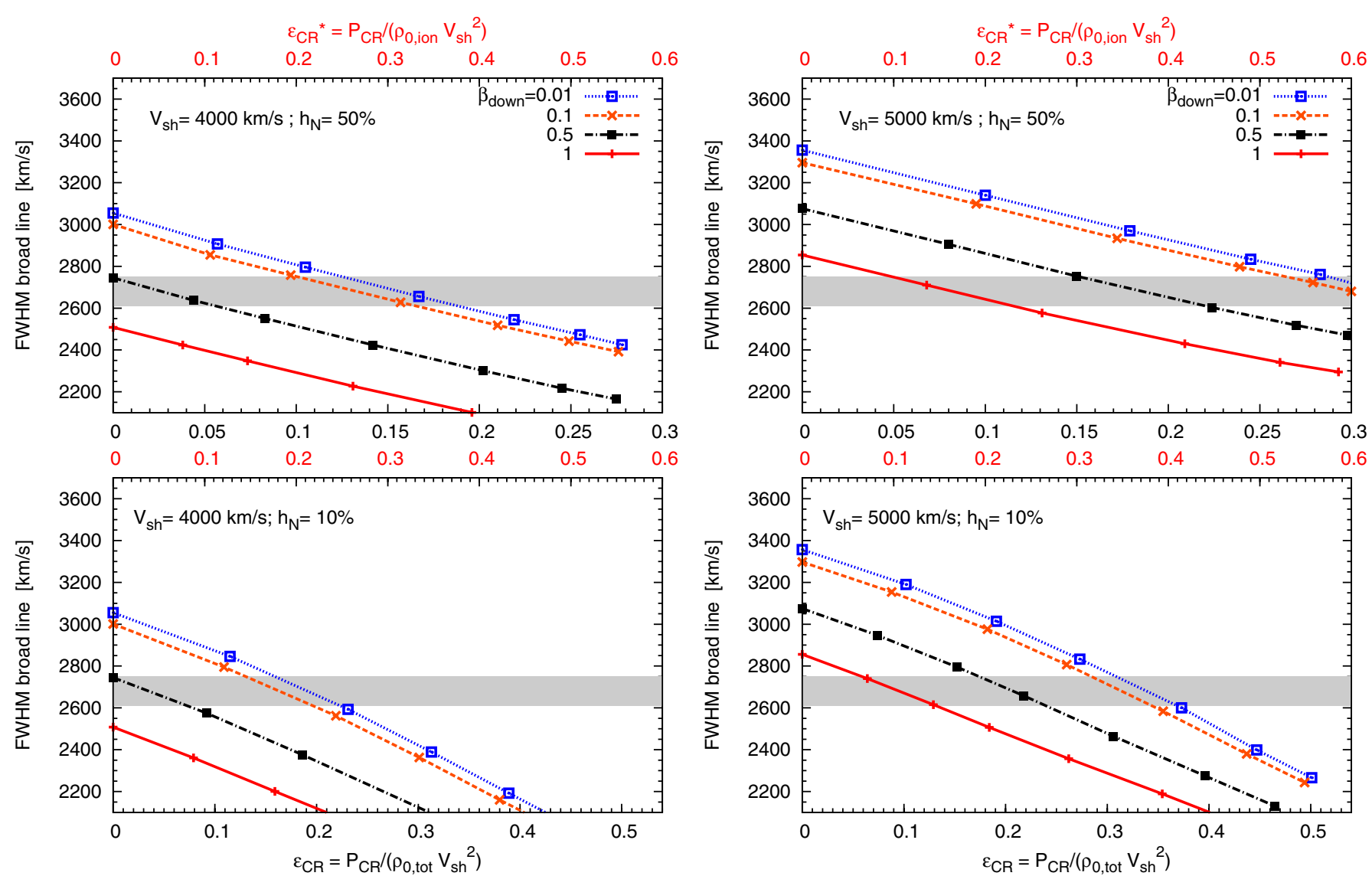

Fig. 3. FWHM of the broad Balmer line as a function of the CR acceleration efficiency. Different lines show different electron-ion equilibrium levels. The plots on the left (right) are obtained for $V_{\mathrm{sh}}=4000 \mathrm{~km} \mathrm{~s}^{-1}\left(V_{\mathrm{sh}}=5000 \mathrm{~km} \mathrm{~s}^{-1}\right)$. The top (bottom) panels refer to neutral fraction $h_{\mathrm{N}}=50 \%\left(h_{\mathrm{N}}=10 \%\right)$. The total gas density is $n_{0}=0.6 \mathrm{~cm}^{-3}$ (Badenes et al. 2008). The shaded area shows the FWHM as measured for the SW shock by Helder et al. (2010) at $1 \sigma$ level.

the measurement would only be compatible with the absence of CR acceleration for a shock speed $\lesssim 3500 \mathrm{~km} \mathrm{~s}^{-1}$.

\section{The narrow line}

A possible way to break the degeneracy between the acceleration efficiency and the electron-ion equilibrium level would be to measure the narrow Balmer line emission from the same region of the shock where the broad line has been observed. In the presence of effective particle acceleration, a precursor is formed upstream of the shock that may lead to a broadening of the narrow line due to $\mathrm{CE}$ reactions between neutrals and ions inside the precursor (see Morlino et al. 2013a, for a discussion). The combination of measurements of the widths of the two components can in principle allow a determination of the CR acceleration efficiency, although it is worth keeping in mind that additional parameters enter the calculation of the narrow line in the presence of CR acceleration: for instance, the maximum energy of accelerated particles and the level of turbulent heating in the precursor. From the observational point of view, Smith et al. (1994) reported the measurement of the FWHM in three different regions, the center and east rim, both having a FWHM of $25 \pm 2 \mathrm{~km} \mathrm{~s}^{-1}$, and the west rim, with a FWHM of $31 \pm 2 \mathrm{~km} \mathrm{~s}^{-1}$. While the first two are compatible with the standard width of the narrow Balmer line in the ISM, the latest is suggestive of a broadening that can be due to the presence of CRs. Moreover, the shape of the line in the west rim shows evidence of non-Gaussian wings, which can also result from the presence of accelerated particles (Morlino et al. 2013a, and discussion below). One should, however, keep in mind that these measurements refer to regions that are spatially different from those where the broad Balmer line has been measured. This is the reason why we concentrated here our attention on the detailed measurements of the shape and intensity of the broad component.

On the other hand, we think it useful to show how the shape of the narrow Balmer line changes depending on the efficiency of particle acceleration and of nonadiabatic heating in the CR precursor. We selected the case with $V_{\text {sh }}=5000 \mathrm{~km} \mathrm{~s}^{-1}$, $n_{\text {tot }}=0.6 \mathrm{~cm}^{-3}, h_{\mathrm{N}}=10 \%$, and $\beta_{\text {down }}=0.5$ as the best suited to illustrating the effect of CRs on the narrow Balmer line. The maximum momentum and the injection parameter are fixed to $p_{\max }=10 \mathrm{TeV}$ and $\xi_{\text {inj }}=3.7$, respectively, so as to have $\epsilon_{\mathrm{CR}}^{*} \simeq 25 \%$, compatible with the FWHM of the broad Balmer line as shown in Fig. 3.

In the top panel of Fig. 5 we plot the predicted shape of the narrow Balmer line for two values $(0$ and 0.4$)$ of the turbulent heating parameter $\eta_{\mathrm{TH}}$ (namely the amount of turbulent magnetic field that is eventually converted to thermal energy of the plasma upstream; see Morlino et al. 2013a). For comparison, we also show the case without CR acceleration. In the bottom panel we plot the FWHM of the narrow Balmer line as a function of $\eta_{\mathrm{TH}}$.

We discuss this panel first. Here we show that the FWHM of the narrow line is very sensitive to the value of $\eta_{\mathrm{TH}}$. On the other hand, the effect produced by adiabatic compression is very weak: in the absence of any nonadiabatic heating $\left(\eta_{\mathrm{TH}}=0\right)$, the FWHM is only 24 , rather than $21 \mathrm{~km} \mathrm{~s}^{-1}$, which is the width corresponding to the upstream temperature of $10^{4} \mathrm{~K}$ in the absence of CR acceleration. 

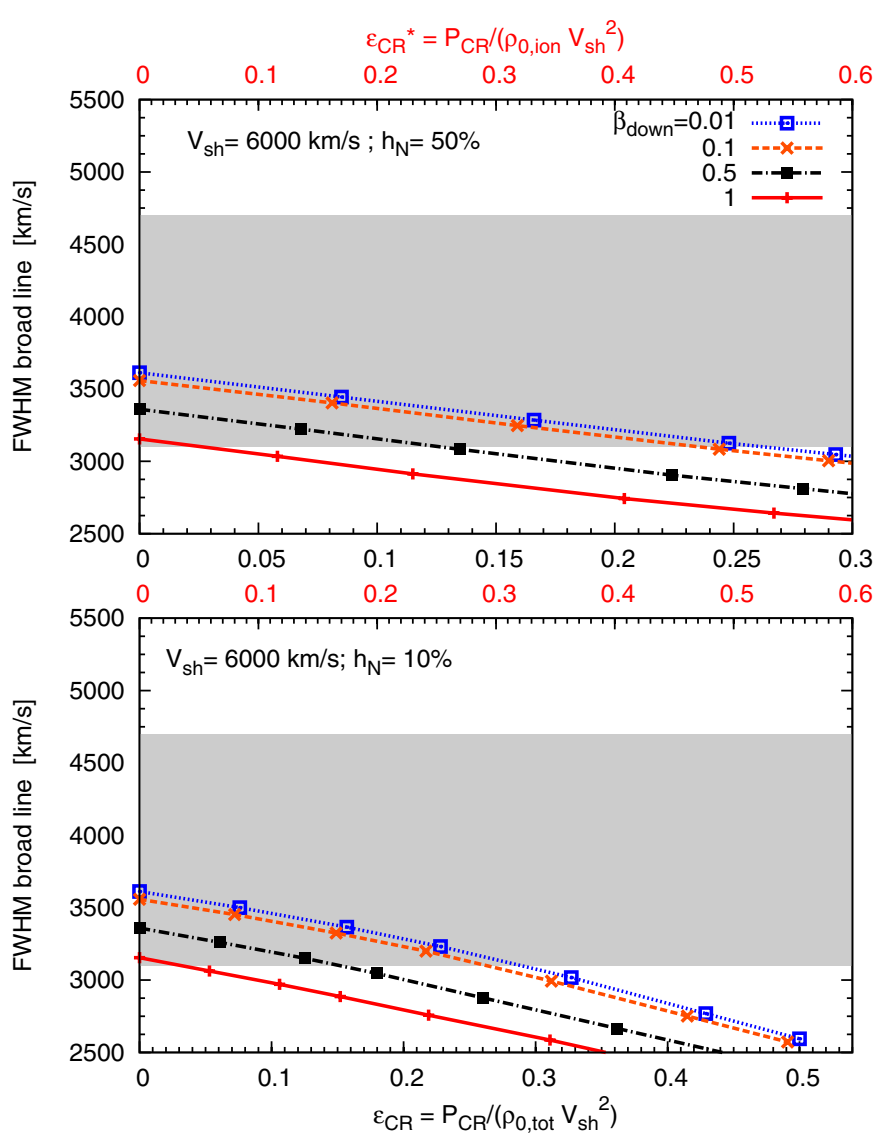

Fig. 4. Same as in Fig. 3 but for shock velocity $V_{\text {sh }}=6000 \mathrm{~km} \mathrm{~s}^{-1}$. Here the shaded area shows the FWHM measured for the NE sector of the shock by Helder et al. (2010) at $1 \sigma$ level.

In the top panel of Fig. 5 we compare the line profile for the case when CR acceleration is absent (FWHM of $21 \mathrm{~km} \mathrm{~s}^{-1}$ ) with a Gaussian profile having the same width. The weak wings that appear at high $v_{x}$ are the result of the neutral return flux discussed by Blasi et al. (2012): this phenomenon is very weak for shock velocity $>3000 \mathrm{~km} \mathrm{~s}^{-1}$. In the same panel we also plot the profile of the narrow Balmer line for $\eta_{\mathrm{TH}}=0$ and 0.4. One can appreciate that in both cases the width of the line increases, more prominently for the larger value of $\eta_{\mathrm{TH}}$. In addition to the broadening however, one can also notice the rise of non-Gaussian wings, which are not due to the neutral return flux (relatively small for large shock velocities) but rather to the CR-induced precursor upstream. A broadening of the narrow Balmer line and the appearance of non-Gaussian wings are the imprint of CR acceleration.

\section{Conclusions}

The formalism proposed by Blasi et al. (2012) and Morlino et al. $(2012,2013 a)$ to describe the process of diffusive particle acceleration at shocks in the presence of neutral hydrogen atoms was applied here to SNR 0509-67.5, for which measurements of the width of the broad Balmer line have recently become available (Helder et al. 2010). Such measurements were carried out on the SW and NE rims of the remnant, returning a FWHM of $2680 \pm 70 \mathrm{~km} \mathrm{~s}^{-1}$ and $3900 \pm 800 \mathrm{~km} \mathrm{~s}^{-1}$, respectively.

For the NE rim, the FWHM of the broad line is fully consistent with standard shock dynamics in the absence of accelerated particles, for a shock velocity of $6000 \mathrm{~km} \mathrm{~s}^{-1}$. For the SW rim, Helder et al. (2010) use $5000 \mathrm{~km} \mathrm{~s}^{-1}$ as a lower limit to the shock velocity, based on a simple model of the dynamics of the
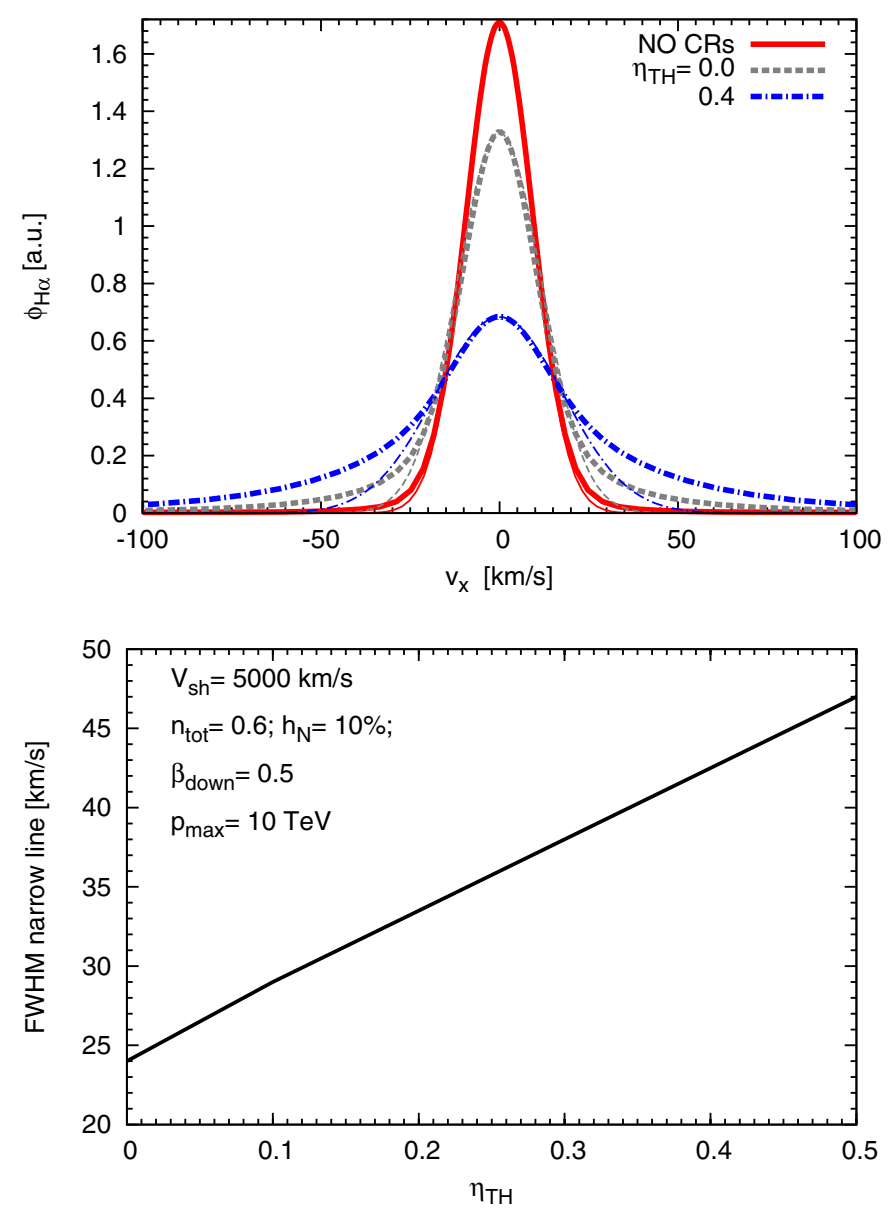

Fig. 5. Top panel: thick lines show the profile of the narrow Balmer line for three cases: no CR acceleration (solid line) and acceleration efficiency $\epsilon_{\mathrm{CR}}^{*}=25 \%$ (i.e. $\epsilon_{\mathrm{CR}}=22 \%$ ) for two different values of the turbulent heating parameter, $\eta_{\mathrm{TH}}=0$ (dashed) and 0.4 (dot-dashed). For all the three cases the thin lines show the corresponding Gaussian having the same FWHM of the narrow Balmer line. Bottom panel: FWHM of the narrow Balmer line as a function of $\eta_{\mathrm{TH}}$, for acceleration efficiency $\epsilon_{\mathrm{CR}}^{*}=25 \%$. For both panels the values of the other parameters are fixed as shown in the label of the bottom panel.

remnant. On the other hand, we showed that the asymmetry between the NE and SW parts of the SNR suggest a somewhat lower velocity for the SW rim. Our calculations show that shock velocities between 3500 and $5500 \mathrm{~km} \mathrm{~s}^{-1}$ are still compatible with the measured angular size of the remnant in the SW region. In the NE, a shock velocity of $\sim 6000 \mathrm{~km} \mathrm{~s}^{-1}$ seems appropriate.

To quantify the impact of the uncertainty in the shock velocity on the estimate of the CR acceleration efficiency, we carried out the calculations of the shock dynamics, of particle acceleration and of Balmer line emission for $V_{\mathrm{sh}}=4000$ and $5000 \mathrm{~km} \mathrm{~s}^{-1}$ in the case of the SW rim and for $V_{\mathrm{sh}}=6000 \mathrm{~km} \mathrm{~s}^{-1}$ for the NE rim. For both rims two values of the neutral fraction at upstream infinity were considered: $h_{\mathrm{N}}=50 \%$ and $h_{\mathrm{N}}=10 \%$.

If the shock speed in the $\mathrm{SW}$ region is $5000 \mathrm{~km} \mathrm{~s}^{-1}$, our calculations show evidence of particle acceleration with efficiency between $\sim 10 \%$ and $\sim 50 \%$, depending on the level of thermal equilibrium between electrons and ions downstream of the shock. The inferred CR acceleration efficiency depends very weakly upon the value of the gas density around the SNR. Lower shock speeds lead to a less clear situation: for $V_{\mathrm{sh}}=4000 \mathrm{~km} \mathrm{~s}^{-1}$, the evidence of CR acceleration appears to be solid only if a low level of thermal equilibrium between ions and electrons is assumed $\left(\beta_{\text {down }} \sim 0.1-0.01\right)$, in which case the CR acceleration 
efficiency is at the level of $\sim 20-30 \%$. In the NE rim, the error bar on the measured value of the FWHM is so large that it is not possible to infer any firm conclusion in terms of particle acceleration.

Measurements of the width of the narrow Balmer line at the same spatial locations where the broad Balmer emission is measured may help resolve the degeneracy between CR acceleration and electron-ion equilibrium level, but such measurements are so far not available for this SNR. However, we also calculated the shape of the narrow Balmer line for the same set of parameters that appear to describe the broad emission, so as to show the predictive power associated with the measurement of the narrow component. We find that the presence of CRs certainly leads to a broadening of the narrow Balmer line, but this effect is rather sensitive to the fraction $\eta_{\mathrm{TH}}$ of turbulent field energy density that is transformed into heating of the upstream plasma. In addition to a larger FWHM of the narrow line (typical widths range between 30 and $50 \mathrm{~km} \mathrm{~s}^{-1}$, to be compared with $21 \mathrm{~km} \mathrm{~s}^{-1}$ expected in the absence of CR acceleration), the presence of a CR-induced precursor leads to the appearance of non-Gaussian wings in the shape of the line, more pronounced for higher values of $\eta_{\mathrm{TH}}$.

Acknowledgements. We are grateful to D. Caprioli for discussions on the topic. This work was partially funded through grant PRIN INAF 2010 and ASTRI.

\section{References}

Amato, E., \& Blasi, P. 2005, MNRAS, 364, L76

Amato, E., \& Blasi, P. 2006, MNRAS, 371, 1251

Badenes, C., Hughes, J. P., Bravo, E., Langer, N. 2007, ApJ, 662, 472
Badenes, C., Hughes, J. P., Cassam-Chenaï, G., \& Bravo, E. 2008, ApJ, 680, 1149

Blasi, P., Morlino G., Bandiera R., Amato, E., \& Caprioli, D. 2012, ApJ, 755, 121 (Paper I)

Borkowski, K. J., Williams, B. J., Reynolds, S. P., et al., 2006, ApJ, 642, L141

Caprioli, D., Blasi, P., Amato, E., \& Vietri, M. 2008, ApJ, 679, 139

Chevalier, R. A., \& Raymond, J. C. 1978, ApJ, 225, L27

Ghavamian, P., Blair, W. P., Sankrit, R., Raymond, J. C., \& Hughes, J. P. 2007, ApJ, 664, 304

Ghavamian, P., Schwartz, S.J., Mitchell, J., Masters, A., \& Laming, J. M. 2013, Space Sci. Rev., in press [arXiv: 1305.6617]

Helder, E. A., Kosenko, D., \& Vink, J. 2010, ApJ, 719, L140; Erratum: 2011, 737, L46

Morlino, G., \& Caprioli, D. 2012, A\&A, 538, A81

Morlino G., Bandiera R., Blasi, P., \& Amato, E. 2012, ApJ, 760, 137 (Paper II)

Morlino G., Blasi, P., Bandiera R., \& Amato, E. 2013a, ApJ, 768, 148 (Paper III)

Morlino G., Blasi, P., Bandiera R., \& Amato, E. 2013b, A\&A, in press, DOI: 10.1051/0004-6361/201322006

Malkov, M.A., \& Drury, L. O’C., 2001, Rep. Prog. Phys., 64, 429

Rakowski, C. E. 2005, Adv. Space Res., 35, 1017

Raymond, J. C., Vink, J., Helder, E. A, \& de Laat, A., 2011, ApJ, 731, L14

Rest, A., Suntzeff, N. B., Olsen, K., et al. 2005, Nature, 438, 1132

Rest, A., Matheson, T., Blondin, S., et al. 2008, ApJ, 680, 1137

Schaefer, B. E., \& Pagnotta, A. 2012, Nature, 481, 164

Seok, J. Y., Koo, B.-C., Onaka, T., et al., 2008, PASJ, 60, S453

Smith, R. C., Raymond, J. C., \& Laming, J. M. 1994, ApJ 420, 289

Truelove, J. K., \& McKee, C. F. 1999, ApJ, 120, 299

Tuohy, I. R., Dopita, M. A., Mathewson, D. S., Long, K. S., \& Helfand, D. J. 1982, ApJ, 261, 473

van Adelsberg, M., Heng, K., McCray, R., \& Raymond, J. C. 2008, ApJ, 689, 1089

Wagner, A. Y., Lee, J.-J., Raymond, J. C., Hartquist, T. W., \& Falle, S. A. E. G. 2009, ApJ, 690, 1412

Warren, J. S., \& Hughes, J. P. 2004, ApJ, 608, 261 